\title{
On Separating Two Simple Polygons by a Single Translation*
}

\author{
G. Toussaint
}

School of Computer Science, McGill University, 805 Sherbrooke Street West, Montreal, Quebec, Canada H3A 2 K6

\begin{abstract}
Let $P$ and $Q$ be two disjoint simple polygons having $n$ sides each. We present an algorithm which determines whether $Q$ can be moved by a single translation to a position sufficiently far from $P$, and which produces all such motions if they exist. The algorithm runs in time $O(t(n))$ where $t(n)$ is the time needed to triangulate an $n$-sided polygon. Since Tarjan and Van Wyk have recently shown that $t(n)=O(n \log \log n)$ this improves the previous best result for this problem which was $O(n \log n)$ even after triangulation.
\end{abstract}

\section{Introduction}

Spurred by developments in spatial planning in robotics, computer graphics, and VLSI layout, considerable attention has been devoted recently to the problem of moving polygons in the plane without collisions [1]-[11]. A typical problem in robotics is the FIND-PATH problem [12], where a robot must determine if an object, modeled as a polygon in the plane, can be moved from a starting position to a goal state without collisions occurring between the object being moved and the obstacles. Much work has been done on the problem of hypothesizing channels through free space when the obstacles are convex polygons [13]. For nonconvex objects the problem is bypassed by considering the convex hulls of the objects to be the objects themselves. Thus a crucial aspect of robotics for the geometric modeling needed for spatial reasoning and spatial planning is the representation and recognition of the possible types of movement allowed by different nonconvex shapes [14]. For a survey of movability problems in computational geometry see [15] and for a survey of the relation of computational geometry to robotics see [16].

\footnotetext{
* This research was supported by NSERC Grant A9293, FCAR Grant EQ-1678, and a Killam Fellowship from the Canada Council.
} 
A robotics problem , nore closely related to the problem considered in this paper is grasping an object with a robot hand. Ignoring several factors such as forces and friction, and severing the hand from the arm leads to some purely geometrical problems. In particular, if we consider only two-dimensional space and model the "hand" and the "object" as two polygons then an interesting geometrical problem consists of determining for a given "hand"-"object" configuration whether the "hand" is truly grasping the "object," i.e., whether the two polygons are interlocked. Several results along these lines are surveyed in [15]. Interesting simplifications occur when the polygons have additional structure [17].

In this paper we develop an algorithm for the problem stated in the abstract. That is, for a given pair of disjoint simple polygons $P$ and $Q$ each having $n$ sides or vertices determine whether $Q$ can be moved by a single translation to a position sufficiently far from $P$ without colliding with $P$ and produce all such motions if they exist. This problem was first considered by Toussaint and Sack [5] who showed that it could be solved in $O\left(n^{2}\right)$ time. Later this result was improved to $O(n \log n)$ time [18], [19]. The approach used in [18] and [19] is via point-location in planar subdivisions [20]. The region outside $P$ but inside the convex hull of $P$ is decomposed into a subdivision such that when a vertex of $Q$ falls in this region its directions of translation can be determined in constant time. However, finding the region in which the query vertex lies takes $O(\log n)$ time. This is done for all vertices of $Q$. The entire procedure is repeated with the roles of $P$ and $Q$ reversed. Finally, the movability of the polygons is determined from the movability of the vertices.

A more difficult problem is that of determining whether $Q$ can be moved by a sequence of translations to a position sufficiently far from $P$ without colliding with $P$, and produce such a motion if it exists. Pollack et al. [21] present an algorithm for solving this problem in time $O\left(n^{2} \alpha\left(n^{2}\right) \log ^{2} n\right)$ where $\alpha(k)$ is the extremely slowly growing inverse Ackermann's function. Since in the worst case $\Omega\left(n^{2}\right)$ translations may be necessary to separate $Q$ from $P$, their algorithm is close to optimal.

In this paper we give an algorithm for solving the single-translation problem in time $O(t(n))$ where $t(n)$ is the time needed to triangulate an $n$-sided polygon. Since Tarjan and Van Wyk [32] have recently shown that $t(n)=O(n \log \log n)$ this represents an improvement over the previous best algorithm which required $O(n \log n)$ time even after triangulation [19].

\section{Geodesic Paths and Relative Convex Hulls}

Let $P=\left(p_{1}, p_{2}, \ldots, p_{n}\right)$ and $Q=\left(q_{1}, q_{2}, \ldots, q_{n}\right)$ be two simple polygons in the plane with nonintersecting interiors. Clearly, the cardinalities of $P$ and $Q$ need not be equal but this assumption simplifies notation. We assume that the polygons are given in standard form, i.e., their vertices, specified in terms of cartesian coordinates, are listed in clockwise order, i.e., the interior always lies to the right of each edge as the polygon is traversed and no three consecutive vertices are 


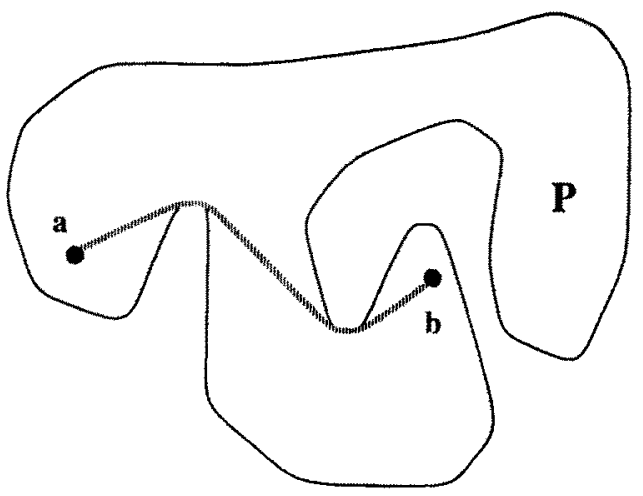

Fig. 1. The geodesic path $\mathrm{GP}(a, b / P)$.

collinear. We say that $P$ and $Q$ are separable under translation (or more succinctly separable) if there exists a direction $\theta$ such that $Q$ can be translated in direction $\theta$ an arbitrary distance without colliding with $P$. By a direction we mean an equivalence class of oriented parallel lines. In some of the concepts to be defined later we use the notion of direction to mean simply an equivalence class of parallel lines. When this is the case we explicitly use the term unoriented direction. Two polygons $P$ and $Q$ collide if at some instant in time, during the motion, their interiors intersect, i.e., $\operatorname{int}(P) \cap \operatorname{int}(Q) \neq$ the null set.

Given a polygon $P$ and two points $a, b \in P$, the shortest path (or geodesic path) between $a$ and $b$ is a polygonal path connecting $a$ and $b$ which lies entirely in $P$ such that the sum of its euclidean edge-lengths is a minimum over all other internal paths. We denote it by $\operatorname{GP}(a, b \mid P)$ where the direction is from $a$ to $b$ (see Fig. 1). Geodesic paths find application in many areas such as image processing [22], operations research [23], visibility problems in graphics [24], and robotics. Recently, Chazelle [25] and Lee and Preparata [23] independently discovered the same $O(n \log n)$ algorithm for computing $\operatorname{GP}(a, b \mid P)$. Both of these algorithms first triangulate $P$ and then find the shortest path in $O(n)$ time. More recently, an algorithm due to ElGindy [26] computes $\operatorname{GP}(a, b \mid P)$ without first triangulating $P$.

Definition. A polygonal circuit is a closed polygonal path without self-propercrossings. (This is a slight generalization of the notion of a simple polygon to allow some vertices and edges to be used more than once.) Thus it makes sense to speak of its interior and exterior [27]. Accordingly, we also refer to this as a weakly-simple polygon.

Definition. The convex hull of $P$ relative to $Q$, denoted by $\mathrm{CH}(P \mid Q)$ is the shortest polygonal circuit (or geodesic circuit) which contains $P$ and excludes $Q$; i.e., $\operatorname{int}(P) \subseteq \operatorname{int}(\mathrm{CH}(P \mid Q))$ and $\operatorname{int}(Q) \subseteq \operatorname{ext}(\mathrm{CH}(P \mid Q))$. Figure 2 illustrates two polygons and $\mathrm{CH}(P \mid Q)$. Figure 3 illustrates a case where the $\mathrm{CH}(P \mid Q)$ is not a simple polygon. We also refer to $\mathrm{CH}(P \mid Q)$ and $\mathrm{CH}(Q \mid P)$ as relative convex hulls. 


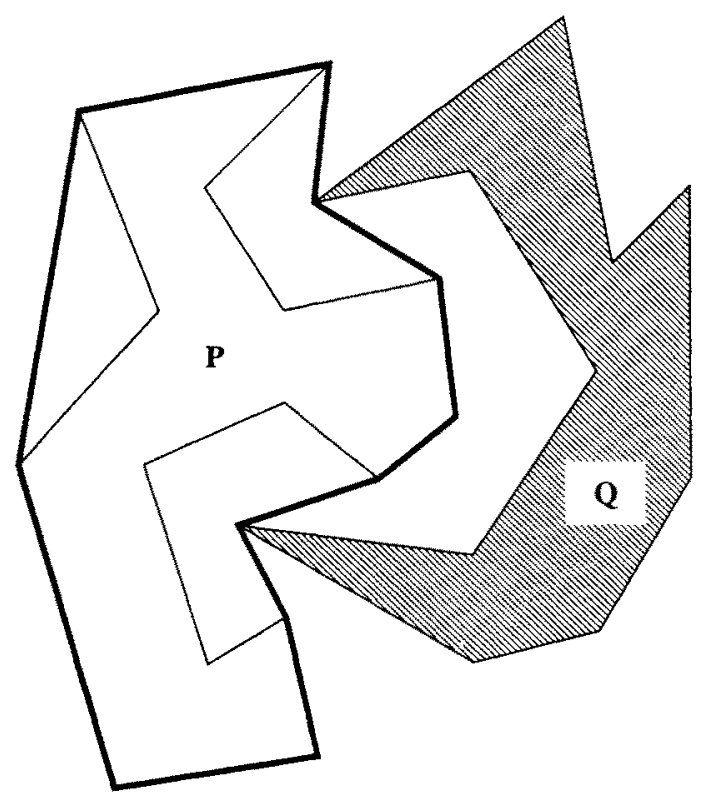

Fig. 2. Hlustrating the relative convex hull $\mathrm{CH}(P / Q)$.

\section{Geodesic Circuits and Separability of Polygons}

In this section we present the main result of this paper: we show that, given two nonintersecting simple polygons $P$ and $Q$, the translation separability problem can be reduced to computing the relative convex hulls of $P$ and $Q$. This result is expressed by Theorem 1 below. First we introduce some more notation.

The sides of polygon $P$, called edges, are denoted by $e_{j}=\left(p_{j}, p_{j+1}\right)$ and are directed from $p_{j}$ to $p_{j+1}$ (indices are modulo $n$ throughout). A chain $C_{i j}(P)=$ $\left(e_{i}, e_{i+1}, \ldots, e_{j-1}\right)$ is a sequence of edges on the boundary of $P$. Similarly, for $Q$ we have $f_{j}=\left(q_{j}, q_{j+1}\right)$ and $C_{i j}(Q)=\left(f_{i}, f_{i+1}, \ldots, f_{j-1}\right)$. A chain $C_{i j}(P)$ is monotonic with respect to direction $\theta$ if the projections of the vertices $p_{i}, p_{i+1}, \ldots, p_{j}$ onto a

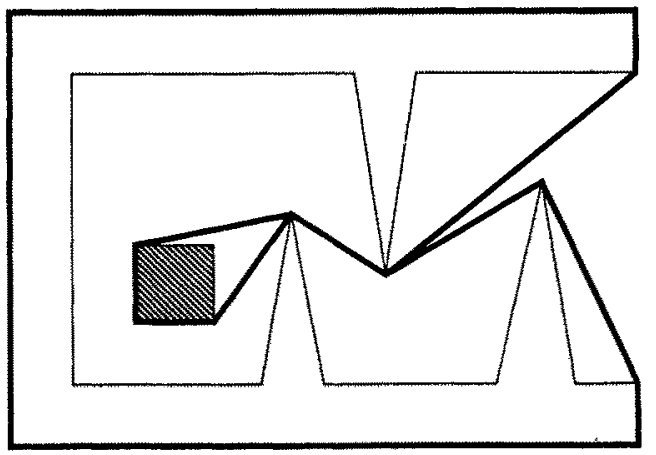

Fig. 3. A case when the relative convex hull is a weakly-simple polygon. 
line $L(\theta)$ in unoriented direction $\theta$ are ordered as the vertices in $C_{i j}(P) . P$ is a monotonic polygon if there exists a line $L(\theta)$ such that the boundary of $P$ can be partitioned into two chains $C_{i j}(P)$ and $C_{j i}(P)$ that are monotonic with respect to $\theta$.

We can now state the main result.

Theorem 1. Two disjoint simple polygons $P$ and $Q$ are separable under translation if, and only if, their relative convex hulls are monotonic polygons.

Before proving Theorem 1 we need a few lemmas.

Definition. Given a simple polygon $P$ and an unoriented direction $\theta$, the visibility hull of $P$ in direction $\theta$, denoted by $\operatorname{VH}(P, \theta)$, is the set obtained by taking the union of $P$ with all line segments $(a, b)$ in direction $\theta$ such that $a, b \in P$. Note that $\operatorname{VH}(P, \theta)$ is monotonic with respect to $\theta+90^{\circ}$. The edges on the boundary of $\mathrm{VH}(P, \theta)$ which are not edges of $P$ specify a set of "pockets" of $\mathrm{VH}(P, \theta)$. See Fig. 4 for an illustration of the visibility hull of $P$ and its "pockets."

Lemma 1 [17]. Two disjoint monotonic polygons $P$ and $Q$ are separable under translation.

Lemma 2. Let $P$ be a polygon monotonic in the unoriented direction $\theta$ and let $a$, $b$ be any two points in $P$. Then the geodesic path $\operatorname{GP}(a, b \mid P)$ is a polygonal chain monotonic with respect to $\theta$.

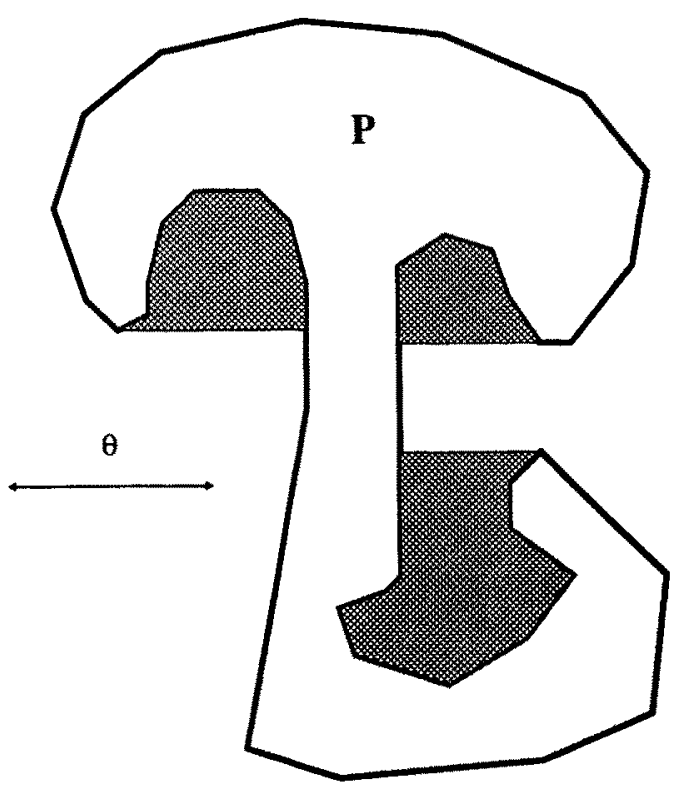

Fig. 4. Illustrating the visibility hull of $P$ in direction $\theta$. 
Proof. Let $\operatorname{GP}(a, b \mid P)$ be nonmonotonic with respect to $\theta$. Without loss of generality assume $\theta$ to be parallel to the $x$ axis and let $a$ have smaller $x$ coordinate than $b$. This implies that there exists a pair of consecutive vertices of $\operatorname{GP}(a, b \mid P)$, say $u, v$, such that $u$ occurs before $v$ on a traversal of $\operatorname{GP}(a, b \mid P)$ from $a$ to $b$, and $v$ has smaller $x$ coordinate than $u$. But since all the vertices of $\operatorname{GP}(a, b \mid P)$, other than $a$ and $b$, coincide with vertices of $P$, it follows from the Jordan Curve Theorem that the boundary of $P$ contains a chain $C_{y}(P)$ which is not monotonic with respect to $\theta$.

Lemma 3 [5]. Two disjoint simple polygons $P$ and $Q$ are separable under translation in unoriented direction $\theta$ if, and only if,

$$
\operatorname{int}[\operatorname{VH}(P, \theta)] \cap \operatorname{int}[\mathrm{VH}(Q, \theta)]=\varnothing .
$$

We are now ready to prove Theorem 1 .

Proof of Theorem 1. [if part] If $\mathrm{CH}(P \mid Q)$ and $\mathrm{CH}(Q \mid P)$ are monotonic, then it follows from Lemma 1 that they can be separated under translation. Now, by definition $\mathrm{CH}(P \mid Q)$ contains $P$ and $\mathrm{CH}(Q \mid P)$ contains $Q$. Therefore, $P$ and $Q$ are separable under translation.

[only if part] We must show that if $P$ and $Q$ are separable then both relative hulls are monotonic. Assume therefore that $P$ and $Q$ are separable in unoriented direction $\theta$. Compute the visibility hulls $\mathrm{VH}(P, \theta)$ and $\mathrm{VH}(Q, \theta)$. From Lemma 3 it follows that their interiors do not intersect. Now construct the relative convex hulls of the visibility hulls $\mathrm{CH}[\mathrm{VH}(P, \theta) \mid \mathrm{VH}(Q, \theta)]$ and $\mathrm{CH}[\mathrm{VH}(Q, \theta) \mid \mathrm{VH}(P, \theta)]$. These relative hulls must also be the relative hulls of $P$ and $Q$, respectively. If this were not the case then the relative hull of, say, $P$, $\mathrm{CH}(P \mid Q)$ would intersect either a pocket of $\mathrm{VH}(P, \theta)$ or a pocket of $\mathrm{VH}(Q, \theta)$. In either case it would imply that $P$ and $Q$ cannot be separated in direction $\theta$, a contradiction. Now, the relative convex hulls of $\mathrm{VH}(P, \theta)$ and $\mathrm{VH}(Q, \theta)$ form

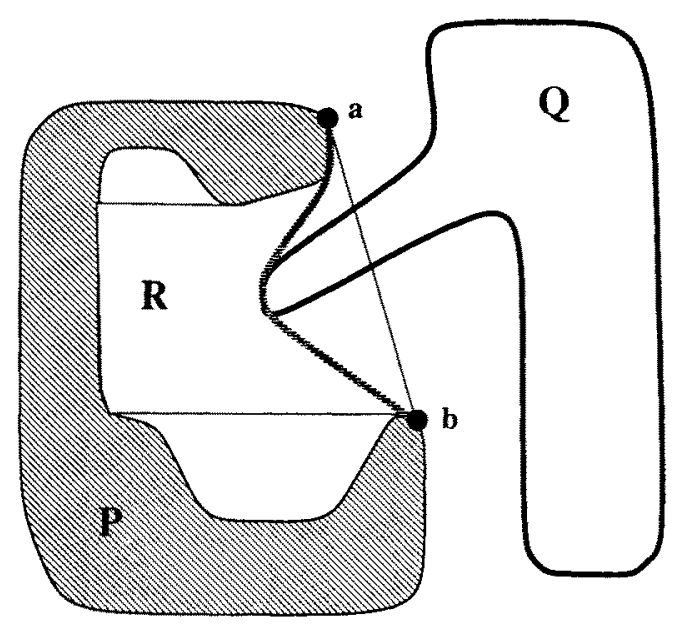

Fig. 5. Illustrating the proof of Theorem 1. 
convex polygons except for the chains lying inside pockets of the convex hulls of $P$ and $Q$, respectively. Consider one such pocket determined by vertices $a$ and $b$ and refer to Fig. 5. The region $R$, where $\mathrm{GP}(a, b \mid P)$ must lie, is a monotonic polygon with respect to $\theta+90^{\circ}$ and from Lemma 2 it follows that $\operatorname{GP}(a, b \mid P)$ is a monotonic chain with respect to $\theta+90^{\circ}$. Since this is true for all such pockets we have that $\mathrm{CH}(P \mid Q)$ and $\mathrm{CH}(Q \mid P)$ are both monotonic with respect to $\theta+90^{\circ}$.

\section{The Algorithm}

Theorem 1 suggests the following algorithm for solving the separability problem stated in the abstract. Compute the relative convex hulls of $P$ and $Q$ and determine whether they are monotonic polygons. With the algorithm of Preparata and Supowit [31] we can determine whether the relative convex hulls are monotonic in $O(n)$ time. Thus the crucial part of the problem is computing the relative convex hulls.

Consider the polygons $P$ and $Q$ in Fig. 6 and the convex hull of $P, \mathrm{CH}(P)$. It is clear that if $Q$ did not intersect $\mathrm{CH}(P)$ then we would have $\mathrm{CH}(P \mid Q)=$ $\mathrm{CH}(P)$. This observation suggests an approach to computing $\mathrm{CH}(P \mid Q)$ by first

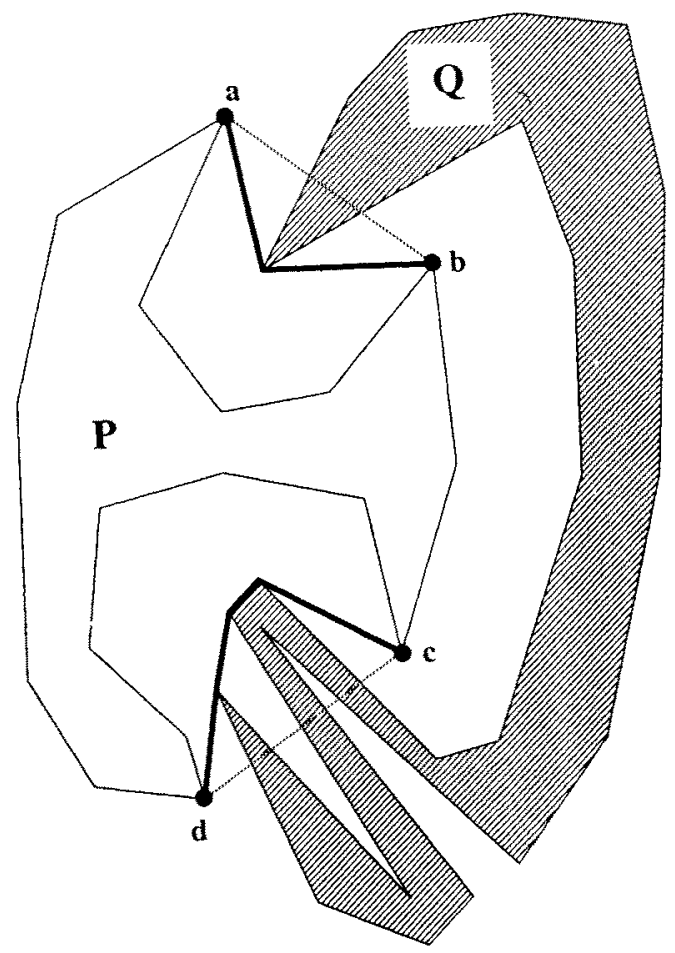

Fig. 6. Illustrating one possible way to compute $\mathrm{CH}(P / Q)$. 
determining $\mathrm{CH}(P)$ and subsequently patching up $\mathrm{CH}(P)$ at those pockets where $Q$ intersects $\mathrm{CH}(P)$ to obtain $\mathrm{CH}(P \mid Q)$. All we need to do is compute the shortest path in each pocket of $\mathrm{CH}(P)$ from the endpoints of the pocket lids (such as $(a, b)$ and $(c, d)$ in Fig. 6) such that the shortest paths separate $P$ from $Q$. However, it is not clear how to patch up all the pockets with a total complexity less than $O(n \log n$ ). If for each pocket (such as the one determined by $c d$ in Fig. 6) we had a list of all the boundary points of $Q$ intersected by line segment $c d$ and, furthermore, if these intersection points were sorted along $c d$, then $O(t(n))$ time would suffice to compute $\mathrm{CH}(P \mid Q)$. A straightforward scan of these intersection points would isolate a simple polygon, call it $R$, inside the pocket of $c d$ in which the geodesic path from $c$ to $d$ is guaranteed to lie. This scan has a complexity linear in $k_{1}$, the number of vertices of $Q$ contained in the pocket of $c d$. The region $R$ can then be triangulated in time $t\left(k_{1}+k_{2}\right)$ where $k_{2}$ is the number of vertices of $P$ contained in the pocket of $c d$. Finally, the geodesic path between $c$ and $d$ can be computed in $O\left(k_{1}+k_{2}\right)$ time. Adding the time taken for all the pockets of $P$ would lead to a complexity of $O(t(n))$. Unfortunately, it is not clear how to obtain all the intersection points in sorted order efficiently. Sorting Jordan sequences in linear time [28] does not appear to help. If for each pocket lid $P$ we apply Jordan sorting to $Q$ this results in an overall complexity of $O\left(n^{2}\right)$.

One way to reduce this complexity is by using the line-segment intersection algorithm of Mairson and Stolf [29]. They have shown that given two sets of $n$ line segments $S_{1}$ and $S_{2}$ such that the elements in each set are pairwise disjoint (their interiors do not intersect), all the intersecting pairs between $S_{1}$ and $S_{2}$ can be reported in $O(n \log n+I)$ time where $I$ is the number of such pairs. Furthermore, for each line segment their algorithm reports all the intersection points in sorted order along the line segment. Now, in general, $I$ can be $O\left(n^{2}\right)$ but in the problem considered here we have an additional structure that can be exploited. In our problem $S_{1}$ consists of the edges of $Q$ and $S_{2}$ consists of the pocket lids of the pockets of $\mathrm{CH}(P)$. Furthermore, these lids form a convex polygon. Therefore each line segment of $S_{1}$ can intersect at most two line segments of $S_{2}$ and therefore $I=O(n)$. Thus this approach solves the separability problem in $O(n \log n)$ time [37]. Although this is much better than $O\left(n^{2}\right)$ it is not an improvement over [19].

We are able to reduce the complexity to $O(t(n))$ by using an additional simple lemma. Refer to Fig. 7.

Definition. A bridge of $\mathrm{CH}(P \cup Q)$ is an edge of $\mathrm{CH}(P \cup Q)$ joining a vertex of $P$ to a vertex of $Q$. An endpoint of a bridge will be called a $P$-endpoint (resp. a $Q$-endpoint) if the endpoint is a vertex of $P$ (resp. $Q$ ).

In general, a bridge $B_{i}$ will connect some vertex $p_{u}$ of $P$ to some vertex $q_{v}$ of $Q$. If $p_{u}$ and $q_{v}$ are the endpoints of bridge $B_{i}$ we highlight this fact by using the notation $p_{u t}$ and $q_{v i}$. If the discussion is independent of the actual values of $u$ and $v$ we use the noiation $p_{\bullet_{i}}$ and $q_{\bullet_{i}}$ to specify the endpoints of bridge $B_{i}$. 
Lemma 4 [30]. Given two nonintersecting simple polygons $P, Q$, the convex hull $\mathrm{CH}(P \cup Q)$ has either zero or two bridges.

Proof. Zero bridges result when one polygon lies in the interior of the convex hull of the other. Consider two consecutive bridges $B_{i}, B_{i+1}$. If $B_{i}$ has a pair of endpoints $\left(p_{\cdot i}, q_{\cdot i}\right)$, then $B_{1+1}$ must have a pair $\left(q_{\bullet_{t+1}}, p_{v_{i+1}}\right)$. Therefore an odd number of bridges is impossible since we would have a chain of one polygon containing vertices of the other polygon. Therefore we can only have an even number of bridges. Now two can occur when $P$ and $Q$ are linearly separable. Assume we have an even number greater than two and consider two of these $B_{1}$ and $B_{2}$. The $P$-endpoints of $B_{1}$ and $B_{2}$ are connected by two chains of $P$ and the $Q$-endpoints by two chains of $Q$. Therefore any other bridge $B_{i}, i>2$, implies $P$ and $Q$ intersect, which is a contradiction.

We are now ready to complete the description of the algorithm. In Case 1, then, we have the $\mathrm{CH}(P \cup Q)$ and two bridges $B_{1}=\left(q_{\bullet_{i}}, p_{.}\right)$and $B_{j}=\left(p_{\cdot j}, q_{. j}\right)$ illustrated in Fig. 7. Note that the two bridges and the chains $C_{{ }_{j} \cdot i}(P)$ and $C_{.,{ }_{j}}(Q)$ define a simple polygon $Z(P, Q)$ which "separates" $P$ and $Q$. The $\mathrm{CH}(P \mid Q)$ is the concatenation of the partial convex hull of $P$ from $p_{\cdot i}$ to $p_{._{j} j}$ and the geodesic path in $Z(P, Q)$ from $p .$, to $p_{._{1}}$. Similarly, the $\mathrm{CH}(Q \mid P)$ is the union of the partial hull of $Q$ from $q_{* j}$ to $q_{* \text {. }}$ and the geodesic path in $Z(P, Q)$ from $q_{*_{i}}$ to

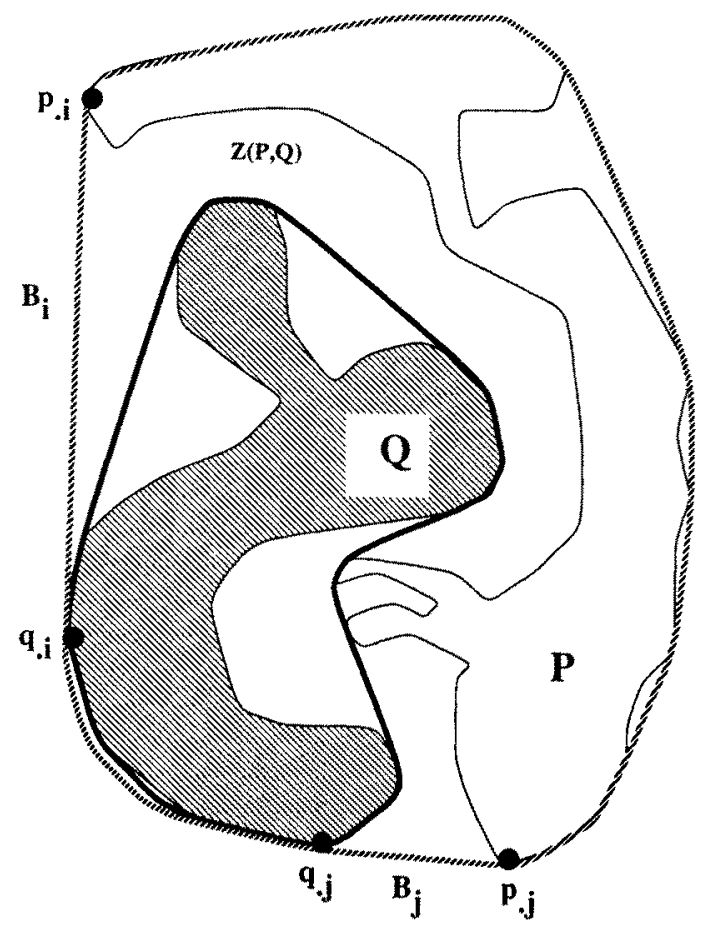

Fig. 7. The convex hull of the union of two disjoint polygons has either zero or two bridges. 


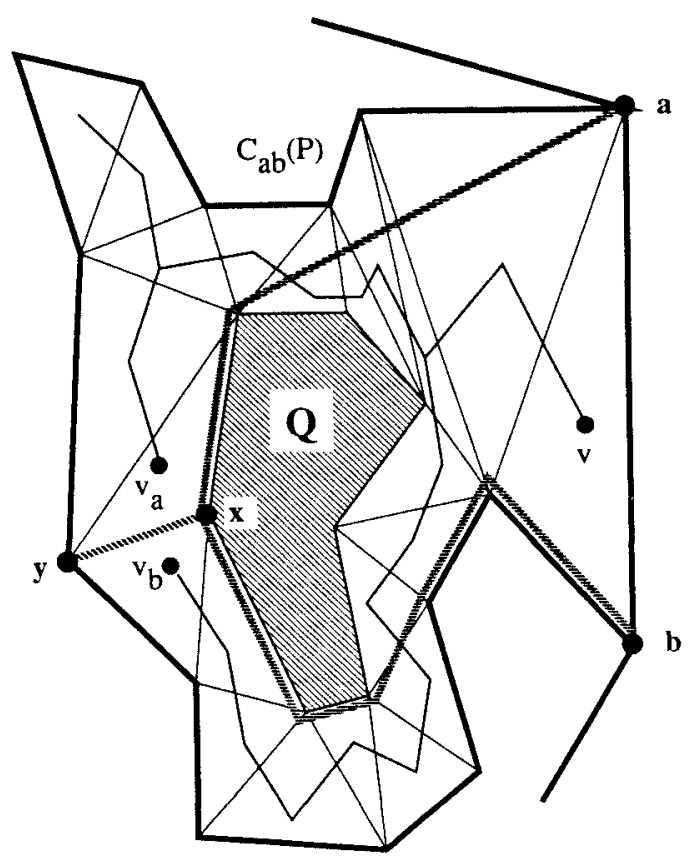

Fig. 8. Illustrating the algorithm when $Q$ lies entirely in the interior of one pocket of $\mathrm{CH}(P)$.

q.y. Since $Z(P, Q)$ is simple, we can triangulate it in $O(n \log \log n)$ time with the algorithm of Tarjan and Van Wyk [32]. Note that all computations other than triangulation are either linear or sublinear. The convex hulls of $P$ and $Q$ can be computed in $O(n)$ time with the algorithm of McCallum and Avis [34] and detecting whether or not they intersect can be done in $O(\log n)$ time with the algorithm of Chazelle and Dobkin [35]. Finally, computing the convex hull of $P \cup Q$ can be done in $O(n)$ time using the "rotating caliper" algorithm of Toussaint [36].

In Case 2, when $Q$ lies entirely in the interior of one pocket of $P$, the situation is slightly more involved. Let $a b$ denote the pocket lid of some pocket $K$, of $P$ and refer to Fig. 8 . The pocket $K_{j}$ is itself a simple polygon determined by line segment $a b$ concatenated with chain $C_{b a}(P)$. In this case the region of interest in which we need to computer geodesic paths is the set-difference of $K$, and the interior of $Q$. This region referred to as $K^{\prime}$, is not a simple polygon but contains a "hole." The first step is to convert $K_{j}^{\prime}$ into a weakly simple polygon $P^{\prime}$ by adding two "copies" of a "connecting bridge" $x y$ between a vertex of $Q$ and a vertex of $C_{a b}(P)$.

Definition. Given a pocket $K_{j}$ of $P$ properly containing polygon $Q$, a connecting bridge between $Q$ and $K_{j}$ is a line segment $(x, y)$ such that (a) $(x, y)$ does not intersect any edge of $K$, or $Q$ except at its endpoints, (b) int $(x, y)$ lies in int $(K$, and in $\operatorname{ext}(Q)$, and (c) one endpoint of $(x, y)$ is a vertex of $Q$ and the other endpoint is a vertex of $C_{a b}(P)$ other than $a$ and $b$. 
Lemma 5. Given two polygons $R_{\text {out }}$ and $R_{\text {in }}$, where $R_{\text {in }}$ is properly contained in $R_{\text {out }}$, with $n$ and $m$ vertices, respectively, a connecting bridge between $R_{\text {in }}$ and $R_{\text {out }}$ can be found in $O(m+n)$ time.

Proof. The proof is straightforward and the details are omitted.

Once a bridge $(x, y)$ between $Q$ and $C_{a b}(P)$ has been found, $P^{\prime}$ can be triangulated and its dual tree $T$ obtained. Three vertices of $T$ play a singular role here. Let $v$ be the node of $T$ associated with the triangle having $(a, b)$ as one of its edges. Let $v_{a}$ and $v_{b}$ be the nodes of $T$ associated with the two triangles that share the connecting bridge $(x, y)$. If $C_{a b}(P)$ is traversed in order starting at $a$, then an ordering of the triangles is induced. Of the two triangles sharing the connecting bridge, the first to be encountered in this ordering corresponds to $v_{a}$, the second to $v_{i}$. Although we cannot be sure that $\mathrm{CH}(P \mid Q)$ must go through a specified point $z$, we do have the following lemma.

Lemma 6. $\mathrm{CH}(P \mid Q)$ must intersect the connecting bridge $(x, y)$.

Proof. By construction, $K_{j}$ is a simple polygon. By definition, $\mathrm{CH}(P \mid Q)$ in $K_{j}$, together with $(a, b)$, is a weakly simple polygon, and in fact can be viewed as the relative convex hull $\mathrm{CH}\left[Q \cup(a, b) \mid C_{a b}(P)\right]$ and therefore must contain $Q$. It follows that any connecting bridge must intersect $\mathrm{CH}(P \mid Q)$.

Lemma 6 now allows us to compute the geodesic path between $a$ and $b$ constrained to pass through the connecting bridge $x y$ in linear time using the algorithms of Chazelle [25] or Lee and Preparata [23]. The algorithms in [25] and [23] compute the geodesic path between two points in a sleeve in linear time. A sleeve is a polygon whose dual tree is a chain. In our problem (see Fig. 8), the shortest path from $v_{a}$ to $v$ in $T$ yields sleeve $S_{a}$. Similarly, the shortest path from $v_{b}$ to $v$ yields sleeve $S_{b}$. Unfortunately, the union of $S_{a}$ and $S_{b}$ where $\mathrm{GP}\left(a, b \mid K_{3}\right)$ must lie is not a simple polygon. However, we can get around this obstacle by embedding $S_{a} \cup S_{b}$ onto a Riemann surface [33] of two levels. We embed $S_{a}$ onto level one and $S_{b}$ onto level two with a ramp at the connecting bridge $x y$ leading from level one to level two thus obtaining a Riemann sleeve. To the algorithms in [23] and [25] the Riemann sleeve so constructed looks just as if it were a regular sleeve. It follows that once $P^{\prime}$ is triangulated $\mathrm{CH}(P \mid Q)$ can be computed in linear time.

All that remains is to compute $\mathrm{CH}(Q \mid P)$. In this case it is easily verified that if we choose a connecting bridge $(x, y)$ such that $x$ is the vertex of $Q$ furthest from the line through $(a, b)$ then $\mathrm{CH}(Q \mid P)$ must traverse $x$. Since the shortest path between $v_{a}$ and $v_{b}$ in $T$ is a chain it follows that $\operatorname{GP}\left(a^{\prime}, b^{\prime} \mid P^{\prime}\right)$ where $a^{\prime}$ and $b^{\prime}$ are any two points lying in the triangles associated with $v_{a}$ and $v_{b}$, respectively, must lie in a regular sleeve. Recall that in triangulating $P^{\prime}$ we first obtained a weakly simple polygon by inserting two copies of $(x, y)$. Let $x_{a}$ and $x_{b}$ denote the two copies of $x$ on the side of $v_{a}$ and $v_{b}$, respectively. It follows that $\mathrm{CH}(Q \mid P)$ is $\operatorname{GP}\left(x_{a}, x_{b} \mid P^{\prime}\right)$ and can be computed as before. 
In summary, we have shown:

Theorem 2. Given two disjoint simple polygons $P$ and $Q$ of $n$ edges each, whether they are separable under translation can be determined in $O(t(n))$ time where $t(n)$ is the time needed to triangulate a polygon with $O(n)$ edges.

If $P$ and $Q$ are separable under translation it means $\mathrm{CH}(P \mid Q)$ and $\mathrm{CH}(Q \mid P)$ are monotonic with respect to some unoriented direction $\theta$. Thus a motion for separation is immediate. Either $P$ or $Q$ can be translated in either of the two oriented directions determined by $\theta+90^{\circ}$ and which of these orientations is valid can be determined in $O(n)$ time. Actually, once the relative convex hulls are available all directions in which $P$ and $Q$ are separable under translation can be computed in linear time. It suffices to know that the wedge of all possible directions is determined by the vertices of $P$ (and $Q$ ), that (1) are contained in $\mathrm{CH}(Q)(\mathrm{CH}(P))$, and (2) that are also vertices of $\mathrm{CH}(P \mid Q)(\mathrm{CH}(Q \mid P))$. The details are omitted.

As a final note we remark that the new approach for solving the translationseparability problem presented here can lead to optimal algorithms if $P$ and $Q$ have an additional structure that allows triangulation of the required regions to be done in linear time. As an example we obtain the following theorems by applying the previous results [37].

Theorem 3. Given two disjoint monotone polygons $P$ and $Q$ of $n$ edges each, all motions that can take $Q$ sufficiently far from $P$ by a single translation can be determined in $O(n)$ time.

Theorem 4. Given two disjoint star-shaped polygons $P$ and $Q$ of $n$ edges each, all motions that can take $Q$ sufficiently far from $P$ by a single translation can be determined in $O(n)$ time.

\section{Conclusion}

In closing we mention some open problems. The convex hull of a simple polygon can be found in linear time [34]. The relative convex hull seems very closely related to the standard convex hull and two polygons do not seem that much worse than one. Does there exist a linear algorithm for computing $\mathrm{CH}(P \mid Q)$ when $P$ and $Q$ are simple polygons? Two polygons may be interlocked under a single translation but not if rotations are allowed. How fast can we determine if two polygons can be separated with rotations? In three dimensions we may define $\mathrm{CH}(P \mid Q)$ for two polyhedra $P$ and $Q$ as the minimum-area surface enclosing $P$ and excluding $Q$. How fast can we compute $\mathrm{CH}(P \mid Q)$ ? Finally, there exists a family of problems that concern the "penetration" of the convex deficiency of $P$ (the union of the pockets) by $Q$. For example, in the design of drugs we encounter the problem of finding a test molecule (modeled as a polyhedron) that will "fit" well "into" the deficiency of a host molecule. Several possibilities exist 
for measuring the degree of "penetration." One such measure might be the fraction of $\mathrm{CH}(P)$ taken up by $\mathrm{CH}(P) \sim \mathrm{CH}(P \mid Q)$, where $\sim$ denotes set difference. An open problem in both two and three dimensions is to determine for $P$ and $Q$ the maximum penetration under translations and rotations of $Q$ without allowing collisions.

\section{Acknowledgment}

The author is grateful to David Avis and Hossam ElGindy for discussions on this topic and to Binay Bhattacharya for the suggestion that the region $Z(P, Q)$ might help in reducing the complexity of this problem. As always, his intuition was unfailing.

\section{References}

1. L. J. Guibas and F. F. Yao, On translating a set of rectangles, Proc. Twelfth Annual ACM Symposium on Theory of Computing, 154-160 (1980).

2. T. Ottman and P. Widmayer, On translating a set of line segments, Computer Vision, Graphics and Image Processing, Vol. 24, 382-389 (1983).

3. G. T. Toussaint, The complexity of movement, Proc. IEEE International Symposium on Information Theory, St. Jovite (September 1983).

4. J.-R. Sack and G. T. Toussaint, Movability of objects, Proc, IEEE International Symposium on Information Theory, St. Jovite (September 1983).

5. G. T. Toussaint and J.-R. Sack, Some new results on moving polygons in the plane, Proc. Robotic Intelligence and Productivity Conference, Detroit, Michigan, 158-163 (November 18-19, 1983).

6. B. Chazelle et al. The complexity and decidability of SEPARATION, Technical Report CS-83-34, University of Waterloo (November 1983).

7. G. T. Toussaint, On translating a set of polyhedra, Proc. Conference on Polyhedra, Smith College, Northampton, Massachusetts (April 6-8, 1984).

8. R. Dawson, On removing a ball without disturbing the others, Mathematics Magazine, Vol. 57, No. 1, 27-30 (January 1984).

9. K. Post, Six interlocking cylinders with respect to all directions, Internal manuscript, University of Eindhoven (December 1983).

10. W. Moser, Research problems in discrete geometry, Department of Mathematics, McGill University (1981).

11. G. T. Toussaint, Some collision avoidance problems between spheres, Proc. International Conference on Systems, Man and Cybernetics, Tucson, Arizona (November 1985).

12. Van-Duc Nguyen, The find-path problem in the plane, A.I. Memo, No. 160, Arificial Intelligence Laboratory, M.I.T. (February 1984).

13. Bruce R. Donald, Hypothesizing channels through free-space in solving the findpath problem, A.1. Memo, No. 736, Artificial Intelligence Labratory, M.I.T. (June 1983).

14. David Marr and Lucia Vaina, Representation and recognition of the movement of shapes, A.I. Memo, No. 597. Artificial Intelligence Laboratory, M.1.T. (October 1980).

15. G. T. Toussaint, Movable separability of sets, in Computational Geometry, G. T. Toussaint, Ed., North-Holland, Amsterdam, 335-375 (1985).

16. S. Whitesides, Computational geometry and spatial planning, in Computational Geometry, G. T. Toussaint, Ed., North-Holland, Amsterdam, 377-427 (1985).

17. G. T. Toussaint and H. ElGindy, Separation of two monotone polygons in linear time, Robotica, Vol. 2, 215-220(1984). 
18. J.-R. Sack and G. T. Toussaint, Translating polygons in the plane, Proc. STACS `85, Saarbrucken (Januáry 1985).

19. J.-R. Sack and G. T. Toussaint, Separability of pairs of polygons through single translations, Robotica, Vol. 5, 55-63 (1987).

20. D. Kirkpatrick, Optimal search in planar subdivisions, SIAM Journal on Computing, Vol. 12, 28-35 (February 1983).

21. R. Pollack, M. Sharir, and S. Sifrony, Separating two simple polygons by a sequence of translations, Discrete and Computational Geometry, Vol. 3, 123-136 (1988).

22. C. Lantuejoul and F. Maisonneuve, Geodesic methods in quantitative image analysis, Pattern Recognition, Vol. 17, 1177-1187 (1984).

23. D. T. Lee and F. P. Preparata, Euclidean shortest paths in the presence of rectilinear barriers, Networks, Vol. 14, 393-410 (1984).

24. G. T. Toussaint, Shortest path solves edge-to-edge visibility in a polygon, Pattern Recognition Letters, Vol. 4, 165-170 (July 1986).

25. B. Chazelle, A theorem on polygon cutting with applications, Proc. 23rd Annual IEEE Symposium on Foundations of Computer Science, 339-349 (1982).

26. H. ElGindy, Hierarchical deccmposition of polygons with applications, Ph.D. thesis, School of Computer Science, McGill University (May 1985).

27. L. M. Kelly, Ed., The Geometry of Metric and Linear Spaces, Springer-Verlag, New York, (1985).

28. K. Hoffman, K. Melhorn, P. Rosenstiehl, and R. E. Tarjan, Sorting Jordan sequences in linear time, Proc. Symposium on Computational Geometry, Baltimore, Maryland, 196-203 (June 1985).

29. L. J. Guibas and J. Stolfi, Notes on computational geometry, Stanford University (1982).

30. B. K. Bhattacharya and G. T. Toussaint, An $O(n \log \log n)$ time algorithm for determining translation separability of two simple polygons, Technical Report No. SOCS-86.1, McGill University (January 1986).

31. F. P. Preparata and K. J. Supowit, Testing a simple polygon for monotonicity, Information Processing Letters, Vol. 12, 161-164 (August 1981).

32. R. E. Tarjan and C. J. Van Wyk, An $O(n \log \log n)$ time algorithm for triangulating simple polygons, SIAM Journal on Computing, in press.

33. J. Kahn, M. Klawe, and D. Kleitman, Traditional galleries require fewer watchmen, SIAM Journal on Algebraic and Discrete Methods, Vol. 4, 194-206 (June 1983).

34. D. McCallum and D. Avis, A linear time algorithm for finding the convex hull of a simple polygon, Information Processing Letters, Vol. 8, 201-205 (1979).

35. B. M. Chazelle and D. P. Dobkin, Detection is easier than computation, Proc. 12th SIGACT Symposium, Los Angeles, California (1980).

36. G. T. Toussaint, Solving geometric problems with the rotating calipers, Proc. MELECON' 83 , Athens (May 1983).

37. G. T. Toussaint, Shortest path solves translation separability of polygons, Technical Report SOCS-85.27, MeGill University (October 1985).

Received July 26, 1986, and in revised form March 16, 1988. 